\title{
Numerical Simulation of Free Seepage Flow on Non-matching Grids
}

\author{
Bin Jiang ${ }^{1}$ and John C. Bruch, Jr. ${ }^{2}$ \\ 1 Department of Mathematics and Statistics, Portland State University, Portland, \\ OR 97207, USA. bjiang@pdx.edu \\ 2 Department of Mechanical and Environmental Engineering and Department of \\ Mathematics, University of California, Santa Barbara, CA 93106, USA. \\ jcb@engineering.ucsb.edu
}

A new domain decomposition technique on non-matching grids for free boundary problems is considered. An iterative DD scheme is used to reduce the original free boundary problem to a sequence of problems on two sub-domains, one of which includes the free boundary and is described by a variational inequality and the other includes the remainder of the problem and is described by a second order partial differential equation. In the sub-domain which contains the free boundary, a fine grid is utilized in order to capture the free boundary more precisely; while in the other sub-domain, a coarse grid is used in order to speed up the computation. At each step of the iteration, two sub-problems are solved by using a Robin boundary condition on the interface.

\section{Introduction}

Both overlapping and non-overlapping domain decomposition (DD) methods have been intensively studied for partial differential equations, see e.g. [Wid92, Lio90, XZ98, MQ89, Den97]. In the last few decades, mathematicians began to apply the overlapping domain decomposition methods to solve variational inequality problems. The basic idea is to split the original domain into several overlapping sub-domains and solve the variational inequality on each sub-domain via data transfer from the common area between those subdomains. [Bru91, Tai03, HZ92] and their references provide many variants of this approach whereas convergence analyses of the algorithms and their application to many problems in different fields are provided.

However, for many practical problems in the engineering and industrial fields, it is much easier and more convenient to split the original domain into two or three non-overlapping sub-domains and then take care of the problems in each sub-domain where the original problem may show different behavior. Recently, a non-overlapping DD method which utilizes a Robin boundary 
condition on the common boundary between these sub-domains was proposed in [JBS05] for the variational inequality problem and the convergence analysis of the DD method was provided.

In this paper, we consider free seepage flow through a dam with a toe drain that can be considered as a variational inequality. We will apply the non-overlapping DD method to decompose the original problem into two subproblems where the partial differential equation is treated in one sub-domain while the variational inequality is treated in the second sub-domain where the free boundary is located. Since our concern is to find the exact location of the free boundary in the second domain, non-matching grids are applied in those sub-domains. In the first sub-domain, a coarse grid is used in order to speed up the computation; while in the second sub-domain which contains the free boundary, a fine grid is utilized in order to capture the free boundary more precisely. At each step of the iteration, two sub-problems are solved simultaneously by using a Robin boundary condition on the common boundary.

This paper is organized as follows. In Section 2, we formulate the seepage problem and apply the non-overlapping DD method to split the original problem into 2 sub-problems. In Section 3, we utilize the non-matching grid technique in those two sub-domains for the discretization of the problem and then apply the finite difference method with projection on the non-matching grids. Numerical results are reported to show the advantage of our new algorithm. In Section 4, a summary of the paper and some future considerations are outlined.

\section{Formulation of the problem}

Many problems involving free boundaries can be reduced to the study of variational inequalities. In [Bru91], the author proposed several domain decomposition methods to split the domain into two or more sub-domains. Then by iterating between these sub-domains he solved the whole problem and found the free boundary. However, these schemes require the solution of one subproblem at one time. Herein, a DD scheme will be used which can solve these two or even more sub-problems simultaneously.

In this paper, we consider a free boundary seepage problem of flow through a porous dam with a toe drain. For simplicity, the soil in the flow field is assumed to be homogeneous and isotropic, capillary and evaporation effects are neglected. In addition, the flow follows Darcy's law:

$$
\vec{q}=-k \nabla\left[\left(\frac{p}{\rho g}\right)+y\right]
$$

where $\vec{q}$ is the velocity vector, $p$ is the pressure, $k$ is the permeability of the soil, $\rho$ is the density of the fluid, $g$ is the gravitational acceleration, and $y$ is the vertical coordinate(positive upward). The seepage velocity has a potential $\phi(x, y)=k\left[\left(\frac{p}{\rho g}\right)+y\right]$. Meanwhile, let $\psi(x, y)$ be the stream function of the 
flow. In this study, the location of the free surface $\Gamma_{0}=\{x, \bar{f}(x)\}$ and the seepage domain $\Omega$ need to be found, see Figure 1. The seepage domain is defined as:

$$
\Omega=\left\{(x, y): 0<x \leq x_{F}, 0<y<\alpha(x) ; x_{F}<x<x_{C}, 0<y<\bar{f}(x)\right\},
$$

where $x_{F}$ and $x_{C}$ are the distances in the $x$-direction to points $F$ and $C$, respectively, and $\alpha(x)$ is the shape function of the dam profile.

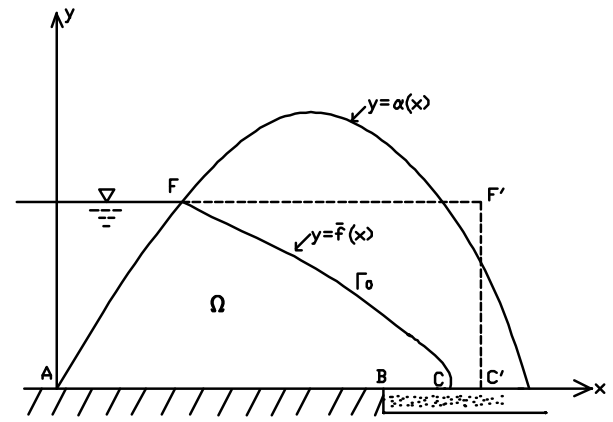

Fig. 1. The seepage problem.

The functions $\phi(x, y)$ and $\psi(x, y)$ are defined on $\bar{\Omega}$ and satisfy the following formulation:

$$
\begin{array}{ll}
\Omega=\{(x, y): & \left.0<x \leq x_{F}, 0<y<\alpha(x) ; x_{F}<x<x_{C}, 0<y<\bar{f}(x)\right\} \\
\phi_{x}-\psi_{y}=0 & \text { in } \Omega \\
\phi_{y}+\psi_{x}=0 & \text { in } \Omega \\
\phi=y_{F} & \text { on } \widehat{A F} \\
\phi=0 & \text { on }[B C] \\
\psi=q & \text { on }[A B] \\
\psi=0 & \text { on } \Gamma_{0} \\
\phi=y & \text { on } \Gamma_{0},
\end{array}
$$

where $y_{F}$ is the height at $F$, and $q$ is the flow rate through the flowfield.

Define $D=\left\{(x, y): 0<x \leq x_{F}, 0<y<\alpha(x) ; x_{F}<x<x_{C^{\prime}}, 0<y<\right.$ $\left.y_{F}\right\}$ and extend $\phi$ and $\psi$ continuously to $\bar{D}$ by setting $\bar{\phi}(x, y)=\phi(x, y)$ in $\bar{\Omega} ;=$ $y$ in $\bar{D}-\bar{\Omega}$ and $\bar{\psi}(x, y)=\psi(x, y)$ in $\bar{\Omega} ;=0$ in $\bar{D}-\bar{\Omega}$.

Next, we can define a new dependent variable $w$ using the Baiocchi transformation on $\bar{D}$ :

$$
w(P)=\int_{\overline{F P}}-\bar{\psi} d x+(y-\bar{\phi}) d y
$$

where $\overline{F P}$ is a smooth path in $D$ joining $F$ to $P$ in $D$. The integration is indeed independent of the path. Then $w \in H^{2}(D) \cap C^{1}(\bar{D})$ satisfies: 


$$
\begin{array}{ll}
\Delta w=\chi_{\Omega} & \text { in } \mathrm{D} \\
w_{y}=y-y_{F} & \text { on } \widehat{A F} \\
w=\left(\frac{q^{2}}{6}\right)+q\left(x_{B}-x\right) & \text { on }[A B] \\
w_{y}=0 & \text { on }[B C] \\
w=0 & \text { in } \bar{D}-\bar{\Omega}\left(\text { also on } \Gamma_{0}\right) \\
w>0 & \text { in } \Omega \quad(w \geq 0 \text { in } \mathrm{D}),
\end{array}
$$

where $\chi_{\Omega}=1$ in $\Omega$ and $\chi_{\Omega}=0$ in $D-\Omega$. Hence,

$$
w(x, y) \geq 0, \quad 1-\Delta w(x, y) \geq 0, \quad w(1-\Delta w)=0 \text { in } D .
$$

If $w$ is found satisfying (2.4), then we can determine $\Omega=\{(x, y) \in D$ : $w(x, y)>0\}$.

It will be seen shortly that if we can properly split $D$ into two nonoverlapping sub-domains, the free boundary is only located in one sub-domain, which makes the original problem simpler. Therefore, the DD method looks promising for this free boundary problem. [Bru91] applied the non-overlapping $\mathrm{D}-\mathrm{N}$ algorithm proposed in [MQ89] to the above problem and the numerical results show that $\mathrm{D}-\mathrm{N}$ algorithm is better than the traditional one-domain finite difference scheme. However, no convergence property of the D-N algorithm can be proven.

Recently, the convergence analysis was provided for a non-overlapping DD method on the uniform meshes with a Robin boundary condition applied to the general free boundary problem represented as variational inequality [JBS05]. In the following we use that DD scheme from [JBS05] to solve the above seepage problem. First, decompose $D$ into subsets $D_{1}=\{(x, y): 0<$ $\left.x<x_{F}, 0<y<\alpha(x)\right\}$ and $D_{2}=\left\{(x, y): x_{F}<x<x_{C^{\prime}}, 0<y<y_{F}\right\}$ with the interface between $D_{1}$ and $D_{2}$ denoted by $\Gamma=\left\{(x, y): x=x_{F}, 0<\right.$ $\left.y<y_{F}\right\}$ in Figure 2. If $w_{1}, w_{2}$ denote the restriction of $w$ in $D_{1}$ and $D_{2}$, respectively, we can write down the following iterative procedure:

Step 1. Initially set $g_{1}^{1}=g_{2}^{1}=0$ on $\Gamma$.

Step 2. Solve the following two sub-problems for $w_{1}^{n}$ and $\left\{w_{2}^{n}, \Omega_{2}^{n}\right\}, n=$ $1,2, \cdots$, respectively:

Problem 1:

$$
\begin{array}{ll}
\Delta w_{1}^{n}=1 & \text { in } D_{1} \\
w_{1}^{n}=\left(\frac{q^{2}}{6}\right)+q\left(x_{B}-x\right) & \text { on }\left[A F_{1}^{\prime}\right] \\
\left(w_{1}^{n}\right)_{y}=y-y_{F} & \text { on } \widehat{A F} \\
w_{1}^{n}+\frac{\partial w_{1}^{n}}{\partial n}=g_{1}^{n} & \text { on } \Gamma .
\end{array}
$$

Problem 2:

$$
\begin{aligned}
& w_{2}^{n}\left(\Delta w_{2}^{n}-1\right)=0 \quad \text { in } D_{2} \\
& w_{2}^{n}=\left(\frac{q^{2}}{6}\right)+q\left(x_{B}-x\right) \quad \text { on }\left[F_{1}^{\prime} B\right] \\
& w_{2 y}^{n}=0 \quad \text { on }\left[B C^{\prime}\right] \\
& w_{2}^{n} \geq 0 \quad \text { in } \overline{D_{2}} \\
& w_{2}^{n}+\frac{\partial w_{2}^{n}}{\partial n}=g_{2}^{n} \quad \text { on } \Gamma \\
& \Omega_{2}^{n}=\left\{(x, y): \quad w_{2}^{n}(x, y)>0\right\} .
\end{aligned}
$$


Step 3. Set

$$
\begin{array}{ll}
g_{2}^{n+1}=2 w_{1}^{n}-g_{1}^{n} & \text { on } \Gamma \\
g_{1}^{n+1}=2 w_{2}^{n}-g_{2}^{n} & \text { on } \Gamma .
\end{array}
$$

Then repeat Step 2 with $n$ replaced by $n+1$. These iterations are stopped when $\max _{\Gamma}\left|w_{1}^{n+1}-w_{1}^{n}\right|<\epsilon$ and $\max _{\Gamma}\left|w_{2}^{n+1}-w_{2}^{n}\right|<\epsilon$, where $\epsilon$ is some fixed error tolerance. Problem 1 and 2 in Step 2 are solved based on the Robin boundary

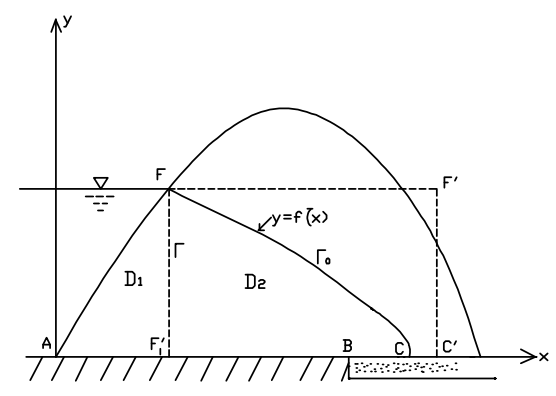

Fig. 2. The domain decomposition.

condition values $g_{1}^{n}$ and $g_{2}^{n}$ which are updated iteratively from their previous value and the $w$ value on the common boundary. This avoids the computation of differentiating $w$ which may reduce the precision.

\section{Non-Matching Grid Discretization and Results}

In this Section, we will utilize a non-matching grid technique to obtain the numerical scheme for the above seepage problem on non-matching grids. At first, we apply the 2nd-order finite difference scheme to $\Delta w$ and obtain the discrete formula for the first equation of (2.6) in $D_{1}$ as follows:

$$
\frac{\left(w_{1}^{n}\right)_{i+1, j}+\left(w_{1}^{n}\right)_{i-1, j}+\left(w_{1}^{n}\right)_{i, j+1}+\left(w_{1}^{n}\right)_{i, j-1}-4\left(w_{1}^{n}\right)_{i, j}}{h_{1}^{2}}=1
$$

where $D_{1}$ is divided into a rectangular mesh with mesh size $\Delta x=\Delta y=h_{1}$, $i, j$ are the row and column mesh point numbers, respectively. The boundary conditions in (2.6) can be discretized by the forward finite difference scheme.

Meanwhile, we can discretize (2.7) in $D_{2}$ in a similar way and obtain

$$
\left(w_{2}^{n}\right)_{i, j}\left(\frac{\left(w_{2}^{n}\right)_{i+1, j}+\left(w_{2}^{n}\right)_{i-1, j}+\left(w_{2}^{n}\right)_{i, j+1}+\left(w_{2}^{n}\right)_{i, j-1}-4\left(w_{2}^{n}\right)_{i, j}}{h_{2}^{2}}-1\right)=0
$$

where $D_{2}$ is divided into a rectangular mesh with mesh size $\Delta x=\Delta y=h_{2}$.

Since our focus is to find the location of the free boundary in $D_{2}$ more precisely, we construct a fine grid in $D_{2}$ and meanwhile construct a coarse grid 
in $D_{1}$ to reduce the computation load there. Therefore, we assume $h_{2}=\frac{1}{2} h_{1}$ throughout our computation, i.e., the grid size of $D_{2}$ is only half of that of $D_{1}$. Because of the different grid sizes in $D_{1}$ and $D_{2}$, the data transfer equations (2.8) between $g_{1}^{n}$ and $g_{2}^{n}$ cannot be discretized naturally. In order to discretize (2.8), we have to approximate $g_{1}^{n}$ with its neighbouring $g_{2}^{n}$ values on $\Gamma$, and vice versa, as shown in Figure 3 . From Figure 3 , we notice that $w_{1, j}$

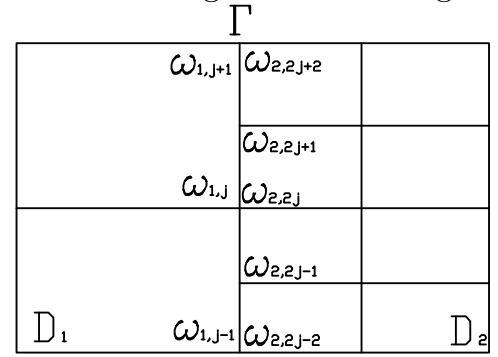

Fig. 3. The nonmatching grids.

is affected by $w_{2,2 j}$. Meanwhile, $w_{2,2 j}$ is affected by $w_{1, j}$ and $w_{2,2 j+1}$ is affected by both $w_{1, j}$ and $w_{1, j+1}$. Therefore, the reasonable data transfer on $\Gamma$ will be $w_{1, j}=w_{2,2 j}$ from $D_{2}$ to $D_{1}$ and $w_{2,2 j}=w_{1, j}$ and $w_{2,2 j+1}=\frac{1}{2}\left(w_{1, j}+w_{1, j+1}\right)$ from $D_{1}$ to $D_{2} \cdot g_{1}$ and $g_{2}$ can be taken care of similarly. Then, (2.8) can be discretized as follows:

$$
\begin{array}{ll}
\left(g_{1}^{n+1}\right)_{j} & =2\left(w_{2}^{n}\right)_{2 j}-\left(g_{2}^{n}\right)_{2 j} \\
\left(g_{2}^{n+1}\right)_{2 j} & =2\left(w_{1}^{n}\right)_{j}-\left(g_{1}^{n}\right)_{j} \\
\left(g_{2}^{n+1}\right)_{2 j+1} & =\frac{1}{2}\left\{\left[2\left(w_{1}^{n}\right)_{j}-\left(g_{1}^{n}\right)_{j}\right]+\left[2\left(w_{1}^{n}\right)_{j+1}-\left(g_{1}^{n}\right)_{j+1}\right]\right\}
\end{array}
$$

The computation is run as follows: at first we scan through $D_{1}$ and $D_{2}$ to solve for $w_{1}^{n}$ and $w_{2}^{n}$ from (3.1) and (3.2) together with the boundary conditions. Then update $g_{1}^{n}$ and $g_{2}^{n}$ on $\Gamma$ from (3.3) and repeat to scan through $D_{1}$ and $D_{2}$ as before. The iteration will stop if the convergence criterion is met.

During the computation, finite difference SOR (Successive over-relaxation) is utilized in $D_{1}$, while in $D_{2}$ which contains the free boundary, finite difference SOR (Successive over-relaxation) with projection is used to make sure the $w_{2}$ value at each point is always non-negative.

Therefore, when applying the SOR in $D_{1},(3.1)$ becomes:

$$
\begin{aligned}
\left(w_{1}^{\left(n+\frac{1}{2}\right)}\right)_{i, j} & =\left(\frac{1}{4}\left(\left(w_{1}^{n}\right)_{i+1, j}+\left(w_{1}^{n}\right)_{i-1, j}+\left(w_{1}^{n}\right)_{i, j+1}+\left(w_{1}^{n}\right)_{i, j-1}-h_{1}^{2}\right)\right. \\
\left(w_{1}^{(n+1)}\right)_{i, j} & =\left(w_{1}^{n}\right)_{i, j}+\bar{\beta}\left(\left(w_{1}^{\left(n+\frac{1}{2}\right)}\right)_{i, j}-\left(w_{1}^{n}\right)_{i, j}\right)
\end{aligned}
$$

where $\bar{\beta}$ is the relaxation parameter.

Similarly, when applying the SOR with projection in $D_{2},(3.2)$ becomes

$$
\begin{aligned}
& \left(w_{2}^{\left(n+\frac{1}{2}\right)}\right)_{i, j}=\frac{1}{4}\left(\left(w_{2}^{n}\right)_{i+1, j}+\left(w_{2}^{n}\right)_{i-1, j}+\left(w_{2}^{n}\right)_{i, j+1}+\left(w_{2}^{n}\right)_{i, j-1}-h_{2}^{2}\right) \\
& \left(w_{2}^{(n+1)}\right)_{i, j}=\max \left(0,\left(w_{2}^{n}\right)_{i, j}+\bar{\beta}\left(\left(w_{2}^{\left(n+\frac{1}{2}\right)}\right)_{i, j}-\left(w_{2}^{n}\right)_{i, j}\right)\right)
\end{aligned}
$$


The flow rate $q$ through the flow field is also unknown a priori. Therefore, in addition to the inner iteration to solve for $w$ with a given $q$, there is also an outer iteration on the $q$ to determine the flow rate. The compatibility condition for the outer iteration (see $[\mathrm{SB} 78])$ is $f(q)=\left(w_{2}\left(x_{F}, y_{F}-\Delta y\right)\right)-\frac{\Delta y^{2}}{2}=0$. In fact, we can set $q_{0}$ and $q_{1}$ to be arbitrary values. Then we use the secant method to determine $q_{2}$ based on $q_{0}$ and $q_{1}$ from (3.6) for the third outer loop, and so on until we reach some $q_{n}$ whose $\left|f\left(q_{n}\right)\right|<\epsilon$.

$$
q_{2}=q_{1}-\frac{q_{2}-q_{1}}{f\left(q_{2}\right)-f\left(q_{1}\right)} f\left(q_{1}\right) .
$$

The example uses the following data: $\alpha(x)=x$ where $0<x<x_{F}, y_{F}=30 \mathrm{ft}$, $x_{F}=30 \mathrm{ft}, x_{B}=60 \mathrm{ft}, h_{1}=0.5 \mathrm{ft}, h_{2}=0.25 \mathrm{ft}, \bar{\beta}=1.25$, and $\epsilon=0.005$.

Figure 4 shows the free boundary obtained by the new DD algorithm. It exactly matches the numerical results from [Bru91]. However, the combination of the new non-overlapping domain decomposition method and the non-matching grid technique generates a better performance than those from [Bru91]. Table 1 shows the required number of iterations for our current algorithm and the algorithm from [Bru91]. We can see that the performance has been improved considerably over [Bru91].

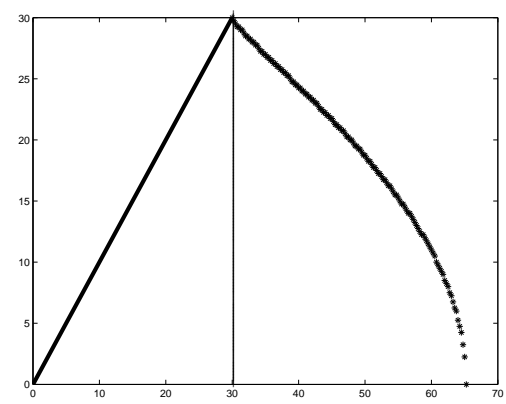

Fig. 4. Free boundary in $D_{2}$.

\begin{tabular}{|c|c|c|}
\hline Outer iteration & Current Algorithm & Algorithm of [Bru91] \\
\hline 1 & 1867 & 2374 \\
\hline 2 & 1024 & 1604 \\
\hline 3 & 1651 & 2088 \\
\hline 4 & 618 & 996 \\
\hline 5 & 109 & 376 \\
\hline 6 & 31 & 156 \\
\hline 7 & 2 & 18 \\
\hline
\end{tabular}

Table 1. Comparison of required number of inner iterations between the two algorithms. 


\section{Conclusion and future directions}

In this paper, we studied a free boundary seepage problem of flow through a porous dam with a toe drain. The characteristic of this problem is that the free boundary is unknown in advance. However, we can determine that the free boundary is located in one of the sub-domains if we can properly split the domain into two or more sub-domains. Then, we can apply the traditional non-overlapping DD method to this problem. Meanwhile, the non-matching grid discretization is utilized on these sub-domains in order to obtain higher resolution of the free boundary in the fine-grid sub-domain while maintaining computational efficiency in the coarse-grid sub-domain.

The promising numerical results motivate us to establish the convergence analysis and error estimates between the numerical solution based on a combination of non-overlapping DD method and non-matching grid discretization and the true solution of the original problem for the general free boundary problem. We will investigate this theoretical issue in the future.

\section{References}

[Bru91] Bruch, Jr., J.C.: Multi-splitting and domain decomposition techniques applied to free surface flow through porous media, Proceedings of the First International Conference on Computational Modeling of Free and Moving Boundary Problems, 3-20 (1991)

[Den97] Deng, Q.: An analysis for a non-overlapping domain decomposition iterative procedure, SIAM J. Sci. Comput., 18, 1517-1525 (1997)

[HZ92] Hoffmann, K., Zou, J.: Parallel algorithms of Schwarz variant for variational inequalities, Numer. Funct. Anal. Optim., 13, 449-462 (1992)

[JBS05] Jiang, B., Bruch, Jr. J.C., Sloss, J.M.: A non-overlapping domain decomposition method for variational inequalities derived from free boundary problems, Numerical Methods Partial Differential Eq., in press (2005)

[Lio90] Lions, P.L.: On the Schwarz alternating method III: A variant for nonoverlapping subdomains, Domain Decomposition Methods for Partial Differential Eq., SIAM, Philadelphia, 202-223 (1990)

[MQ89] Marini, L., Quarteroni, A.: A relaxation procedure for domain decomposition methods using finite element, Numer. Math., 55, 575-598 (1989)

[SB78] Sloss, J.M., Bruch, Jr., J.C.: Free surface seepage problems, J. Eng. Mech. Div. Proc., ASCE, 104, 1099-1111 (1978)

[Tai03] Tai, X.: Rate of convergence for some constraint decomposition methods for nonlinear variational inequalities, Numer. Math., 93, 755-786 (2003)

[Wid92] Widlund, O: Some Schwarz methods for symmetric and non-symmetric elliptic problems, Proceedings of the Fifth International Conference on Domain Decomp. Methods for Partial Differential Eq., 19-36 (1992)

[XZ98] Xu, J., Zou, J.: Some non-overlapping domain decomposition methods, SIAM Review, 40, 857-914 (1998) 